\title{
What constitutes a 'Good' recovery outcome in post-acute Guillain-Barré syndrome? Results of a Nationwide Survey of post-acute GBS sufferers in the United Kingdom
}

\author{
I. Davidson ${ }^{\mathrm{a}}, \mathrm{C}$. Wilson ${ }^{\mathrm{a}}, \mathrm{T}$. Walton ${ }^{\mathrm{b}}$, S. Brissenden ${ }^{\mathrm{b}}$, M. Campbell ${ }^{\mathrm{a}}$ and L. McGowan ${ }^{\mathrm{a}}$ \\ ${ }^{\mathrm{a}}$ School of Nursing, Midwifery and Social Work, University of Manchester, University Place, Manchester; and ${ }^{\mathrm{b}}$ Central Manchester \\ Foundation Trust, currently located in Trafford General Hospital Intermediate Neuro Rehabilitation Unit, Urmston, Manchester, UK
}

\section{Keywords:}

disability, fatigue, Guillain-Barré Syndrome, national survey

Received 7 May 2009

Accepted 3 November 2009
Background: Eighty percent of people with Guillain-Barré Syndrome (GBS) are said to achieve 'good' outcome. 'Good' outcome has been defined as either of the top two scores $(0=$ Healthy, $1=$ minor symptoms or signs, able to run) on a 7-point ordinal scale called the $F$-score. This assessment of 'good' outcome appears to be an arbitrary benchmark. This study is the first assessment of the differences in outcome between post-acute GBS sufferers reporting these scores. It attempts to compare the physical and emotional differences between respondents reporting ' 0 ' and ' 1 ' on the $F$-Score.

Methods: A postal survey was administered to respondents through the UK Guillain-Barré Syndrome Support Group's national database and included items relating to general patient data, general mobility, F-Score, Hospital Anxiety and Depression Scale, SF 36 and Fatigue Severity Scale.

Results: One thousand five hundred and thirty-five members were surveyed, and of $884 / 1535$ (58\%) questionnaires were returned. Results indicate significant differences between those scoring ' 0 ' on the $F$-Score and those scoring ' 1 ' in the post-acute phase in terms of anxiety, depression, physical functioning, fatigue and wheelchair use on discharge.

Conclusions: Significantly poorer outcomes for those scoring ' 1 ' on the F-Score suggest that only those scoring '0' should constitute a 'good' outcome in GBS.

\section{Background}

Guillain-Barré Syndrome affects 1.3-2 people per 100000 population worldwide each year [1]. Despite its relative rarity, it can have serious consequences for those affected [2]. Initial treatment is principally pharmaceutical (normally plasma exchange or intravenous immunoglobulins (IVIg) [3,4]. Severity is such that $20-30 \%$ require ventilation [5].

In the post-acute phase, rehabilitation (especially physiotherapy) features highly [6,7]. The degree to which recovery is achieved post-nadir is multifactorial and may be a function of the condition's natural history, timely drug treatment and rehabilitation, although the relative contribution of each is unclear. Recovery is measured by means of a hierarchical 7-point ordinal

Correspondence: I. Davidson, School of Nursing, Midwifery and Social Work, University of Manchester, University Place, Manchester, UK (tel.: +0161 306 7637; fax: +0161 306 7707;

e-mail: ian.davidson@manchester.ac.uk) scale known as the $F$-Score (or Hughes scale) [8] (Table 1). Using the F-Score, Bernsen et al. [9] offer a means of categorizing the functional outcome of people post- Guillain-Barré Syndrome (GBS) (Table 1) where 'good' outcome is defined as having an $F$-Score of $<2$ which equates to 'minor neurological signs and symptoms'[9].

It is possible that $80 \%$ of sufferers achieve a favourable or 'good' outcome $[10,11]$ at 2 years postnadir [12]. However, in people classified as having a 'good' outcome, symptoms such as fatigue may persist [13] in as many as $80 \%$ of cases [12]. Little work has been conducted on the residual effects of GBS within the subcategory of 'good' recovery and so current categorization of 'good' or otherwise appears arbitrary.

This study aimed to analyse data for people with an ostensibly 'good' recovery following GBS by comparing those reporting their current state as 'healthy' $(F$-score $=0)$ with those stating that they have minor symptoms but who are able to run $(F$-score $=1)$. 


\begin{tabular}{ll}
\hline F-Score $[8]$ & Severity[9] \\
\hline $0=$ healthy & Minor neurological signs \\
$1=$ minor symptoms or signs, able to run & and symptoms \\
$2=$ able to walk $>5 \mathrm{~m}$ without assistance but unable to run & Moderate recovery \\
$3=$ able to walk $>5 \mathrm{~m}$ with assistance & Severe residual signs \\
$4=$ bed or chair bound & \\
$5=$ requiring assisted ventilation for at least part of the day & \\
$6=$ dead & \\
\hline
\end{tabular}

Table 1 Relationship between the $F$-Score and grading system for severity

\section{Method}

One thousand five hundred and thirty-five members of the Guillain-Barré Syndrome Support Group were sent the survey as part of a routine mailing of the Group's newsletter in January 2007. The questionnaire incorporated five areas relating to their condition including demographic and clinical data, information on physical condition and three standardized questionnaires to assess general health status using the SF-36 [14], psychological functioning using the Hospital Anxiety and Depression Scale (HADS) [15] and symptoms of fatigue using the Fatigue Severity Scale (FSS) [12]. Ethical approval for the study was obtained from the local university ethics committee.

The questionnaire was distributed to all members of the group with a covering letter explaining that those with chronic inflammatory demyelinating polyneuropathy (CIDP) should not answer the questionnaire (there was no way of screening out CIDP sufferers from the database). No other limits for inclusion were made. There was no further direct contact with the volunteers but after 6 months, a notice was placed on the Group's website encouraging members to participate but no further reminders were made, and data collection continued for a period of 1 year.

\section{Statistical analysis}

The analysis was restricted to participants aged 18 or over who had $F$-scores of $0-1$ and were walking independently without walking aids. Characteristics and scores on validated measures were compared for those with $F$-scores of ' 0 ' and those with $F$-scores of ' 1 ' using Pearson's chi-square test for dichotomous variables and the Mann-Whitney $U$-test for other (mostly skewed) variables.

Logistic regression models were fitted with relevant explanatory variables to estimate their individual association with the binary outcome $F$-score. Three variables were identified as being theoretically important predictors of the F-score: mean FSS score, SF-36 physical function and regained previous level of mobility prior to GBS diagnosis. These and variables which had $P$-values less than or equal to a conservative $P$-value of 0.20 were included in multivariable models to aid exploration. The variables were investigated for multicollinearity before being included in multivariable logistic regression models to estimate their joint association with the $F$-score. These models included the following variables: mobility on leaving hospital [with two categories: pushed in a wheelchair and independently walking (with or without walking aids)], severe fatigue, regained previous level of mobility prior to GBS diagnosis, mean FSS score, SF-36 physical function, SF-36 role limitation (physical), SF-36 role limitation (mental), SF-36 social function, SF-36 energy and vitality, SF-36 pain, SF-36 health perceptions, HADS anxiety and HADS depression.

\section{Results}

A total of 884 questionnaires were returned (58\% response rate) of which 742 were completed. The 1535 included an unknown number of patients with CIDP, who were asked not to complete the questionnaire; many of those returning uncompleted questionnaires stated that they had indeed been diagnosed with CIDP. The number of respondents aged 18 or over who reported an $F$-Score of ' 0 ' (healthy, 136 respondents) or ' 1 ' (minor symptoms, 101 respondents) was 237 which represents $27 \%$ of all respondents.

Of the analysis sample, $118(50 \%)$ were men, the median age was 62 [interquartile range (IQR) 49-69], the median time since diagnosis was 8 years (IQR 4-13.3), and the median age at diagnosis was 52 (IQR 39.9-61.2).

\section{Comparison of characteristics by F-score group}

Table 2 summarizes the characteristics of those who scored ' 0 ' (Healthy) and ' 1 ' (minor symptoms) on the $F$-Score. The two groups were similar in age and gender, but those in the healthy group had a longer time since diagnosis $(P=0.041)$. The two groups were also similar in length of hospital stay (Fig. 1) and admittance to ICU, but those reporting minor symptoms had a longer stay on ICU (Fig. $2, P=0.018$ ) and were 
Table 2 Characteristics of those scoring ' 0 ' (healthy) and ' 1 ' (minor symptoms but able to run) on the $F$-Score

\begin{tabular}{|c|c|c|c|c|}
\hline & Healthy $(N=136)$ & $\begin{array}{l}\text { Minor symptoms } \\
\text { (able to run) }(N=101)\end{array}$ & $\begin{array}{l}\text { Test statistic for } \\
\text { difference }\end{array}$ & $P$-value \\
\hline \multicolumn{5}{|l|}{ Background } \\
\hline \multicolumn{5}{|l|}{ Age (years) } \\
\hline Median (IQR) & $62.5(49-70)$ & $61(49-68)$ & $\mathrm{M}-\mathrm{W} \mathrm{Z}=0.65$ & 0.519 \\
\hline \multicolumn{5}{|l|}{ Gender: male } \\
\hline Frequency $(\%)$ & $68(50.0 \%)$ & $50(49.5 \%)$ & $\chi^{2}=0.01$ & 0.940 \\
\hline \multicolumn{5}{|c|}{ Time since diagnosis (years) } \\
\hline Median (IQR) & {$[N=135] 8.9(5.0-13.8)$} & {$[N=99] 7.0(2.5-12.8)$} & $\mathrm{M}-\mathrm{W} \mathrm{Z}=2.04$ & 0.041 \\
\hline \multicolumn{5}{|l|}{ Hospital stay } \\
\hline \multicolumn{5}{|c|}{ Duration of stay in hospital (days) } \\
\hline Median (IQR) & {$[N=126] 42(21-70)$} & {$[N=95] 35(21-84)$} & $\mathrm{M}-\mathrm{W} \mathrm{Z}=0.15$ & 0.880 \\
\hline \multicolumn{5}{|l|}{ Admitted onto ICU } \\
\hline Frequency $(\%)$ & {$[N=132] 65(49.2 \%)$} & {$[N=97] 42(43.3 \%)$} & $\chi^{2}=0.79$ & 0.373 \\
\hline \multicolumn{5}{|c|}{ Duration of stay on ICU (days) } \\
\hline Median (IQR) & {$[N=64] 16(6.25-28)$} & {$[N=42] 28(14-42)$} & $\mathrm{M}-\mathrm{W} \mathrm{Z}=2.37$ & 0.018 \\
\hline \multicolumn{5}{|c|}{ Required assisted ventilation } \\
\hline Frequency $(\%)$ & {$[N=131] 45(34.4 \%)$} & {$[N=98] 35(35.7 \%)$} & $\chi^{2}=0.05$ & 0.830 \\
\hline \multicolumn{5}{|c|}{ Being pushed in wheelchair on leaving hospital } \\
\hline Frequency $(\%)$ & {$[N=134] 9(6.7 \%)$} & {$[N=100] 19(19.0 \%)$} & $\chi^{2}=8.20$ & 0.004 \\
\hline \multicolumn{5}{|l|}{ Current health status } \\
\hline \multicolumn{5}{|c|}{ Physical condition at your worst (score) } \\
\hline Median (IQR) & {$[N=133] 5(5-6)$} & {$[N=99] 5(5-6)$} & $\mathrm{M}-\mathrm{W} \mathrm{Z}=1.18$ & 0.237 \\
\hline \multicolumn{5}{|l|}{ Severe fatigue } \\
\hline Frequency $(\%)$ & $30(22.1 \%)$ & {$[N=97] 46(47.4 \%)$} & $\chi^{2}=16.57$ & $<0.001$ \\
\hline \multicolumn{5}{|c|}{ Not having regained previous level of mobility prior to GBS diagnosis } \\
\hline Frequency $(\%)$ & {$[N=134] 17(12.7 \%)$} & {$[N=99] 49(49.5 \%)$} & $\chi^{2}=38.00$ & $<0.001$ \\
\hline
\end{tabular}

[Numbers of responses are shown for variables with missing values].

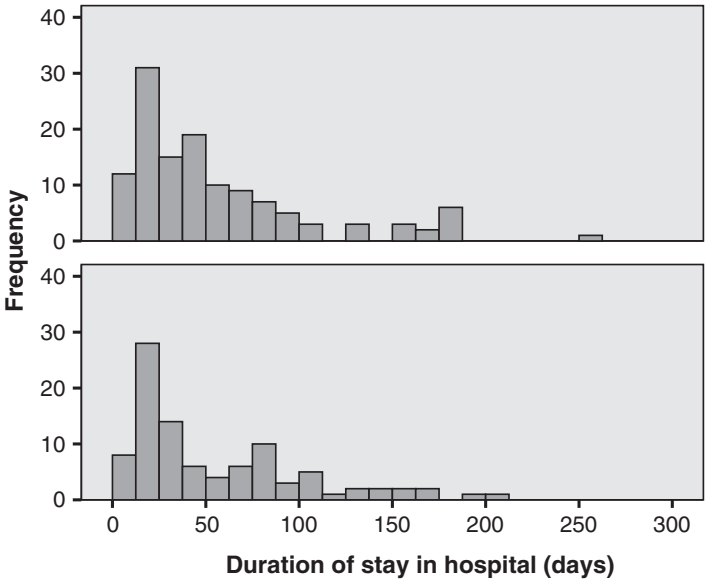

Figure 1 Duration of stay in hospital (days) for those scoring ' 0 ' (healthy) and ' 1 ' (minor symptoms but able to run) on the $F$-Score.

more likely to leave hospital being pushed in a wheelchair $(P=0.004)$.

The two groups reported a median of 5 for $F$-score at worst, and similar percentages reported a need for assisted ventilation. However, those reporting minor symptoms were more likely to suffer severe fatigue $(P<0.001)$. Only $13 \%$ of those in the healthy group reported not regaining their previous level of mobility



Figure 2 Duration of stay in intensive care unit (ICU) (days) for those scoring ' 0 ' (healthy) and ' 1 ' (minor symptoms but able to run) on the $F$-Score.

prior to their diagnosis of GBS compared with $50 \%$ in the group with minor symptoms $(P<0.001)$.

\section{Comparison on validated measures by F-score group}

Table 3 summarizes the scores on the validated measures for those who scored '0' (Healthy) and ' 1 ' 
Table 3 Scores on validated measures for those scoring ' 0 ' (healthy) and ' 1 ' (minor symptoms but able to run) on the $F$-Score

\begin{tabular}{|c|c|c|c|c|}
\hline Scores on validated measures & Healthy $(N=136)$ & $\begin{array}{l}\text { Minor symptoms } \\
\text { (able to run) }(N=101)\end{array}$ & $\begin{array}{l}\text { Test statistic for } \\
\text { difference M-W Z }\end{array}$ & $P$-value \\
\hline \multicolumn{5}{|l|}{ Mean FSS } \\
\hline Median (IQR) & $3.2(2.1-4.8)$ & {$[N=97] 4.8(4.0-5.9)$} & 6.02 & $<0.001$ \\
\hline \multicolumn{5}{|l|}{ SF-36 } \\
\hline \multicolumn{5}{|l|}{ Physical function } \\
\hline Median (IQR) & {$[N=135] 90(80-100)$} & {$[N=100] 76.4(65-90)$} & 4.46 & $<0.001$ \\
\hline \multicolumn{5}{|l|}{ Role limitation (physical) } \\
\hline Median (IQR) & {$[N=134] 100(75-100)$} & {$[N=100] 75(25-100)$} & 4.06 & $<0.001$ \\
\hline \multicolumn{5}{|l|}{ Role limitation (mental) } \\
\hline Median (IQR) & {$[N=133] 100(100-100)$} & {$[N=100] 100(33-100)$} & 3.73 & $<0.001$ \\
\hline \multicolumn{5}{|l|}{ Social function } \\
\hline Median (IQR) & $88.9(88.9-88.9)$ & {$[N=100] 88.9(66.7-88.9)$} & 2.79 & $<0.001$ \\
\hline \multicolumn{5}{|l|}{ Energy and vitality } \\
\hline Median (IQR) & {$[N=135] 65(50-75)$} & {$[N=100] 50(35-65)$} & 4.89 & $<0.001$ \\
\hline \multicolumn{5}{|l|}{ Pain } \\
\hline Median (IQR) & $88.9(77.8-100)$ & {$[N=100] 77.8(66.7-88.9)$} & 5.28 & $<0.001$ \\
\hline \multicolumn{5}{|l|}{ Health perceptions } \\
\hline Median (IQR) & $77(63.3-90)$ & $62(42-77)$ & 5.31 & $<0.001$ \\
\hline \multicolumn{5}{|l|}{ HADS } \\
\hline \multicolumn{5}{|l|}{ Anxiety } \\
\hline Median (IQR) & {$[N=132] 4(2-7)$} & {$[N=98] 6(3.8-10)$} & 2.52 & 0.012 \\
\hline \multicolumn{5}{|l|}{ Depression } \\
\hline Median (IQR) & {$[N=134] 1.5(1-4.3)$} & {$[N=99] 4(2-6)$} & 4.90 & $<0.001$ \\
\hline
\end{tabular}

[Numbers of responses are shown for variables with missing values].

(minor symptoms) on the $F$-Score. For all measures, there was a significant difference between the two groups in the distributions, if not in the median values $(P<0.001$ for all measures except for HADS anxiety score with $P=0.012$ ).

\section{Regression analysis}

Table 4 shows the results of the logistic regression modelling: 218 of the 237 participants had complete data on the variables included in the models. For those scoring ' 1 ' on the $F$-Score (minor symptoms), the adjusted odds of having left hospital in a wheelchair were 4.5 times higher $(P=0.016)$, whilst the odds of regaining a previous level of mobility prior to the GBS diagnosis were three times higher $(P=0.010)$. Again for those in the minor symptoms group, the adjusted odds of having severe fatigue were one-third lower $(P=0.072)$, but this was attributed to the presence of the most important discriminator between the groups, mean FSS score $(P=0.001)$. The unadjusted odds ratio for severe fatigue was 3.19.

Amongst the SF-36 measures remaining in the model, those in the minor symptoms group may have worse pain scores $(P=0.004)$ and worse health perception scores $(P=0.018)$.
Table 4 Associations with $F$-scores of ' 0 ' (healthy) and ' 1 ' (minor symptoms but able to run) using multivariable logistic regression $(N=218)$

\begin{tabular}{|c|c|c|c|}
\hline & $\begin{array}{l}\text { Number of } \\
\text { participants }\end{array}$ & $\begin{array}{l}\text { Adjusted } \\
\text { OR }(95 \% \text { CI })\end{array}$ & $P$-value \\
\hline \multicolumn{4}{|l|}{ Hospital stay } \\
\hline \multicolumn{4}{|c|}{ Being pushed in wheelchair on leaving hospital } \\
\hline No & 184 & 1.00 (reference) & \\
\hline Yes & 22 & $4.50(1.32-15.35)$ & 0.016 \\
\hline \multicolumn{4}{|l|}{ Current health status } \\
\hline \multicolumn{4}{|l|}{ Severe fatigue } \\
\hline No & 140 & 1.00 (reference) & \\
\hline Yes & 66 & $0.35(0.11-1.10)$ & 0.072 \\
\hline \multicolumn{4}{|c|}{ Regained previous level of mobility prior to GBS diagnosis } \\
\hline Yes & 149 & 1.00 (reference) & \\
\hline No & 57 & $3.02(1.30-7.04)$ & 0.010 \\
\hline \multicolumn{4}{|c|}{ Scores on validated measures } \\
\hline Mean FSS score & 206 & $1.83(1.27-2.64)$ & 0.001 \\
\hline SF-36 physical function & 206 & $1.02(1.00-1.04)$ & 0.096 \\
\hline SF-36 pain & 206 & $0.97(0.95-0.99)$ & 0.004 \\
\hline SF-36 health perceptions & 206 & $0.98(0.96-1.00)$ & 0.018 \\
\hline
\end{tabular}

\section{Discussion}

The average age of onset of GBS is typically between $47.7-54.3$ years $[10,12,16]$. Estimates vary in terms of incidence with a ratio of women to men of 1:1.1-2.1[10]. Although the average age of the current sample 
(median: men 62 years women 61 years) is higher than those reported in previous studies, the median time since diagnosis was 8 years, and the median age at diagnosis was 52. Around half were men, which is at the lower end of incidence in reported studies. Whilst this would suggest that these data could be justifiably compared to other study groups by age and gender, it should be noted that the current data only apply to those considered 'good outcome' and not the full spectrum of severity reported in other studies. As such, it is difficult to say whether the results in this sample could be considered typical of GBS. However, because $80 \%$ of sufferers are expected to achieve a 'good' outcome at 2 years post-nadir [10-12], there is no reason to suspect this subsample of the whole cohort is not typical of those reporting an $F$-Score of ' 0 ' and ' 1 '.

Fletcher et al. [5] have identified prognostic indicators for those requiring ventilation for GBS, which include age and length of time on ICU. In this present study, time spent on ICU showed a statistically significant difference between those scoring ' 0 ' (healthy) and those scoring ' 1 ' (minor symptoms) on the $F$-score (medians 16 and 28 days, respectively, $P=0.018$ ). This suggests that despite there being no significant difference between the groups in terms of their $F$-Scores at worst (both scoring a median of $5, P=0.237$ ), the need to be admitted onto ICU $(P=0.373)$ and whether or not artificial ventilation was required $(P=0.830)$, it was possible that the group with minor symptoms has been deemed clinically more severe. This nuance may have been lost given the nature of the $F$-score but was evidently detected by clinical staff at the time of the episode. This may suggest that current outcome measures lack the sensitivity to detect small but clinically significant changes in clinical status and supports the assertion by Chio et al. [10] that there is insufficient understanding of the condition's prognostic factors. Although more sensitive outcome measures have been developed [17], it is possible that most indicative outcomes will only be detected, intuitively or otherwise, by expert practitioners.

\section{Fatigue}

Fatigue is a highly disabling symptom $[10,11]$ commonly reported in GBS [11-13]. Severe fatigue has been reported in as many as $80 \%$ of cases with an $F$-Score range of between 1 and 4 in one patient cohort at the post-acute phase (5.2 years) [12]. Mean FSS score was the strongest discriminator in this present analysis, with those scoring ' 1 ' (minor symptoms) having significantly higher scores $(P=0.001)$ and a higher incidence of 'severe fatigue' $(P<0.001)$ (a mean FSS of at least five [11]). The existence of severe fatigue in 76 of $203(37 \%)$ seems very high in this sample, particularly given the indicative outcome of 'good' and the average length of time since diagnosis (8 years). This underlines the importance of this symptom as a lasting problem in the condition.

Fatigue is not merely a single symptom but also contributes to lower levels of well-being [11]. Results from this present study demonstrate that the 'minor symptoms' group had not only significantly worse scores for fatigue but also health-related quality of life and anxiety and depression.

The relationship between depression and physical illness is complex, and whilst depressed mood may contribute to the development and progression of some illnesses, physical illness can in turn make depression quite probable[18]. In this cohort, the number of respondents with depression and/or anxiety prior to developing GBS is unknown, and neither is it clear how many may have developed psychological distress in response to the aftermath of GBS.

However, from our results, it can be seen that the 'minor symptoms' group were more likely to exhibit raised anxiety and depression scores compared to those in the 'healthy' group. This may be linked to perceived severity of their condition.

Research into other illnesses, such as post-myocardial infarction, has found that although depression and fatigue are highly correlated, there remain a group of people who suffer fatigue without depression [19]. It is possible that those suffering the aftermath of GBS have a similar profile. Interventions applied to fatigued people whether in the form of self-help [20] or external interventions [11,21,22] may assist in improving not only fatigue, but other problems such as anxiety and depression. A reduction in fatigue severity of $20 \%$ has been reported following physical training in post-acute GBS, which appears also to improve levels of anxiety and depression [11].

\section{Physical functioning \& pain}

Multivariable analysis shows that whilst the possibility of those scoring ' 1 ' to report re-establishment of their previous level of functioning is less compared to those scoring ' 0 ', they also experience significantly more pain. Because the $F$-Score is primarily a measure of mobility, it is unsurprising that those scoring ' 1 ' will have a lower level of mobility than those scoring ' 0 ', but the finding that they will also experience more pain may provide an explanatory contributing factor for this.

There is little doubt that pain is associated with GBS [23-25], but the extent to which this is a function of the specific neurological condition itself, musculo-skeletal dysfunction or a combination of both is unclear [26,27]. 
Higher levels of pain in this cohort are associated with lower levels of mobility (poorer $F$-Score), in which case pain could be a barrier to the achievement of an optimal physical outcome.

Moulin et al. [27] suggest that although pain is common particularly in early GBS, intensity diminishes markedly over 24 weeks. It has been shown that in other neuropathies, such as Charcot-Marie-Tooth, there appears to be a clear link between pain and disability [28]. It could reasonably be suggested that the possibility of increased experience of pain especially on movement would make engagement in physical activity less regardless of whether the origin is primarily physical $[26,27]$ or as a result of psychological factors such as catastrophic thinking [29].

Regardless of the cause of pain, the extent to which it shows ostensible diminution with time is interesting, given that the comparison between the two scores shows that those scoring ' 0 ' (Healthy) have had a longer period of time since diagnosis than those scoring ' 1 ' (Table 2 , median values $=9$ vs. 7 years, respectively, $P=0.041)$. Although this is not supported by the regression analysis, possibly because of the low level of significance in the between-group analysis, it could indicate that physical improvement, reconditioning or compensatory (avoidance) strategies may continue over a long period of time. The extent to which this might be a function of pain is unclear.

This notion of prolonged improvement is supported by Fletcher et al. [5], and Bernsen et al. [30] note that $21 \%$ of their cohort reported improvement up to $2.5-$ 6.5 years after onset. This is in line with the results of the present study. It is unlikely that associated debilitation with age is a factor in this observation as there is no significant difference in age between those scoring ' 1 ', and those scoring ' 0 '.

Although length of hospital stay in hospital was similar between the groups, results of the multivariable regression analysis show that respondents with 'minor symptoms' had a greater chance of been pushed in a wheelchair at the point of discharge $(P=0.016$, OR 4.5) than the 'healthy' group. This possibly indicates that the patients with 'minor symptoms' were discharged earlier in their recovery. Reasons for discharge are many and varied but issues such as the rate of recovery, personal motivation, level of home support, limited hospital bed-space and access to rehabilitation post-discharge may be influential.

\section{Limitations of study}

Recall remains a limitation of retrospective selfadministered questionnaires, so data inaccuracy cannot be discounted. The sample is also taken from support group membership which does not represent the entire population of sufferers and so the sample profile may not be truly representative of those currently experiencing the effects of post-acute GBS. Furthermore, a response rate of $58 \%$, whilst good, leaves room for doubt about the representativeness of this cohort.

\section{Conclusion}

To our knowledge, it is the largest survey of this subgroup of GBS sufferers, providing the opportunity for further studies to verify the results and should be considered as a starting point for a more in-depth and overdue debate about the long-term aftermath of GBS. This article has discussed the differences between two groups which together comprise the commonly accepted definition of a 'good' outcome in GBS. These results have shown that the groups are distinctly different in significant ways, and it recommends that these should not be grouped together but instead only an $F$-Score of ' 0 ' or 'Healthy' should be considered as a 'good' outcome. Given the relative homogeneity of the incidence of this condition worldwide, it would be advisable to replicate this study in other countries to verify the underlying trends identified in this present cohort. Areas of further investigation could also consider the causes of pain in GBS and factors influencing the decision to discharge patients from hospital following GBS.

\section{Acknowledgements}

The School of Nursing, Midwifery and Social Work for their financial support. Andy and Maggie Leitch on behalf of the GBS Support Group for assisting in the distribution of the questionnaire to their membership. Lisa Newington for assistance with data entry and cleaning.

\section{References}

1. Hughes R, Swan A, Raphael J, Annane D, Van Koningsveld $\mathrm{R}$, van Doorn PA. Immunotherapy for Guillain-Barré syndrome: a systematic review. Brain Advance Access 2007; March: 1-13.

2. Hughes RA, Cornblath DR. Guillain-Barre syndrome. [Review] [173 refs]. Lancet 2005; 366: 1653-1666.

3. Plasma Exchange/Sandoglobulin Guillain-Barré Syndrome Trial Group. Randomised trial of plasm exchange, intravenous immunoglobulin, and combined treatments in Guillain-Barré syndrome. Lancet 1997; 349: 225-230.

4. van der Meché F, Schmitz P. A randomised trial comparing intravenous immune globulin and plasm exchange in Guillain-Barré syndrome. Dutch Guillain-Barré Study Group. N Engl J Med 1992; 326: 1123-1129. 
5. Fletcher D, Lawn N, Wolter T, Wijdicks E. Long-term outcome in patients with Guillain-Barré Syndrome requiring mechanical ventilation. Neurology 2000; 54: 2311-2315.

6. Davidson I, Wilson C, Walton T, Brissenden S. Physiotherapy and Guillain-Barré Syndrome: results of a National Survey. Physiotherapy 2009; 95: 157-163.

7. Lennon S, Koblar R, Hughes J, Goeller J, Riser A. Reasons for persistent disability on Guillain-Barre syndrome. Clin Rehabil 1993; 7: 1-8.

8. Hughes J, Newsom-Davis J, Perkin G, Pierce J. Controlled trial of prednisolone in acute polyneuropathy. Lancet 1978; 2: 750-753.

9. Bernsen R, de Jager A, Schmitz P, van der Meché F. Residual physical outcome and daily living 3 to 6 years after Guillain-Barre Syndrome. Neurology 1999; 53: 409410.

10. Chio A, Cocito D, Leone M, Giordana M, Mora G, Mutani R. Guillain-Barre syndrome: a prospective, population-based incidence and outcome survey. Neurology 2003; 60: 1146-1150.

11. Garssen MP, Bussmann JB, Schmitz PI, et al. Physical training and fatigue, fitness, and quality of life in GuillainBarre syndrome and CIDP. Neurology 2004; 63: 23932395.

12. Merkies IS, Schmitz PI, Samijn JP, van der Meche FG, van Doorn PA. Fatigue in immune-mediated polyneuropathies. European Inflammatory Neuropathy Cause and Treatment (INCAT) Group. Neurology 1999; 53: 1648 1654.

13. Garssen MP, Blok JH, van Doorn PA, Visser GH. Conduction velocity distribution in neurologically wellrecovered but fatigued Guillain-Barre syndrome patients. Muscle Nerve 2006; 33: 177-182.

14. Brazier J, Harper R, Jones N, et al. Validating the SF-36 health survey questionnaire: new outcome measure for primary care. Br Med J 1992; 305: 160-164.

15. Zigmond A, Snaith R. The hospital anxiety and depression scale. Acta Psychiatr Scand 1983; 67: 361-370.

16. Rees J, Thompson R, Hughes R. Epidemiological study of Guillain-Barré syndrome in south east England. J Neurol Neurosurg Psychiatry 1998; 64: 74-77.

17. Graham R, Hughes R. A modified peripheral neuropathy scale: the overall neuropathy limitations scale. J Neurol Neurosurg Psychiatry 2006; 77: 973-976.
18. Steptoe AE. Depression and Physical Illness. Cambridge: Cambridge University Press, 2009.

19. McGowan L, Dickens C, Percival C, Douglas J, Tomenson $\mathrm{B}$, Creed $\mathrm{F}$. The relationship between vital exhaustion and depression in $1^{\text {st }}$ MI patients. J Psychosom Res 2004; 57: 2-179.

20. Wearden AJ, Riste L, Dowrick C, et al. Fatigue Intervention by Nurses Evaluation - the FINE Trial A randomised controlled trial of nurse led self-help treatment for patients in primary care with chronic fatigue syndrome: study protocol. [ISRCTN74156610]. BMC Med 2006; 4: 9.

21. Pitetti K, Barrett P, Dilawer Abbas B. Endurance exercise training in Guillain-Barré Syndrome. Arch Phys Med Rehabil 1993; 74: 761-765.

22. Walton T, Vincent M, Richards J, Davidson I. Usefulness of digital gait analysis for assessing patients with GuillainBarré Syndrome. Int $J$ Ther Rehabil 2005; 12: 388-393.

23. Karavatas SG. The role of neurodevelopmental sequencing in the physical therapy management of a geriatric patient with Guillain-Barre syndrome. Top Geriatr Rehabil 2005; 21: 133-135.

24. Meythaler J. Rehabilitation of Guillain-Barré Syndrome. Arch Phys Med Rehabil 1997; 78: 872-879.

25. Teunissen LL, Eurelings M, Notermans NC, Hop JW, van GJ. Quality of life in patients with axonal polyneuropathy. J Neurol 2000; 247: 195-199.

26. Kogos J, Richards JS, Banos J, et al. A descriptive study of pain and quality of life following Guillain-Barre syndrome: one year later. J Clin Psychol Med Settings 2005; 12: $111-116$

27. Moulin DE, Hagen N, Feasby TE, Amireh R, Hahn A. Pain in Guillain-Barre syndrome. Neurology 1997; 48: 328-331.

28. Padua L, Cavallaro T, Pareyson D, Quattrone A, Vita G, Schenone A. Charcot-Marie-Tooth and pain: correlations with neurophysiological, clinical, and disability findings. Neurol Sci 2008; 29: 193-194.

29. Sullivan MJL, Lynch ME, Clark AJ. Dimensions of catastrophic thinking associated with pain experience and disability in patients with neuropathic pain conditions. Pain 2005; 113: 310-315.

30. Bernsen RA, de Jager AE, Schmitz PI, van der Meche FG. Long-term impact on work and private life after Guillain-Barre syndrome. J Neurol Sci 2002; 201: 13-17. 